\title{
Single-Field Non-Mydriatic Fundus Photography for Diabetic Retinopathy Screening: A Systematic Review and Meta-Analysis
}

\author{
Jiying Hu ${ }^{a}$ Ruiting Chen ${ }^{a}{\text { Ying } \mathrm{Lu}^{\mathrm{a}} \text { Xiaoyan Dou }}^{\mathrm{b}}$ Baikang Ye ${ }^{\mathrm{b}}$ \\ Zhiming Cai ${ }^{\mathrm{a}}$ Zuhui Pu ${ }^{\mathrm{a}}$ Lisha Mou ${ }^{\mathrm{a}}$ \\ a Shenzhen Evidence-Based Medicine Center, Institute of Translational Medicine, Shenzhen University Health \\ Science Center, Shenzhen University School of Medicine, First Affiliated Hospital of Shenzhen University, Shenzhen \\ Second People's Hospital, Shenzhen, PR China; ${ }^{b}$ Department of Ophthalmology, Shenzhen University School of \\ Medicine, First Affiliated Hospital of Shenzhen University, Shenzhen Second People's Hospital, Shenzhen, PR China
}

\section{Keywords \\ Non-mydriatic fundus photography · Diabetic retinopathy · Diagnostic effectiveness}

\begin{abstract}
Purpose: Single-field non-mydriatic fundus photography (NMFP) has been used to detect diabetic retinopathy (DR) in many studies; however, its value in a general clinical setting has not been established. Here we performed a meta-analysis to evaluate its diagnostic effectiveness. Method: We systematically searched PubMed, EMBASE, and Cochrane databases for candidate studies published through May 19, 2018. A random-effect model was used to calculate the diagnostic indicators including the sensitivity, specificity, positive likelihood ratio (PLR), negative likelihood ratio (NLR), diagnostic odds ratio (DOR), area under the curve (AUC), and 95\% confidence intervals. Results: Ten prospective studies were ultimately included. The pooled sensitivity, specificity, PLR, NLR, and DOR were $0.68,0.94,11.2,0.34$ and 33 , respectively. The AUC was 0.88 . Subgroup analysis showed that single-field NMFP had a respective sensitivity and specificity of 0.73 and 0.91 when compared to standard 7-field mydriatic stereoscopic photography (7SF), and 0.54 and 0.98 when compared to slit-lamp biomicroscopy as reference standard.
\end{abstract}

Conclusions: Single-field NMFP is inadequate to detect DR. Additionally, it showed higher sensitivity and lower specificity when 7SF was used as reference standard, as compared to slit-lamp biomicroscopy, suggesting that different reference standards used in DR screening might have affected the diagnostic results.

(c) 2019 S. Karger AG, Basel

\section{Introduction}

With the rapid growth of the obese population, the incidence and prevalence of diabetes are rising in both developed and developing countries [1,2]. Diabetic retinopathy (DR) is one of the most common microvascular complications of diabetes, and it is a leading cause of blindness [3]. Early detection and treatment of DR can help prevent visual impairment and effectively reduce costs associated with severe visual impairment. Therefore, screening for DR is a cost-effective method in the management of this disease [4-6]. There is a critical need to establish a universally accepted optimal DR screening method in clinical practice.

A variety of techniques have been developed to detect and classify DR, including direct and indirect ophthalmos- (c) 2019 S. Karger AG, Basel

E-Mail karger@karger.com

www.karger.com/ore
Zuhui Pu and Lisha Mou

Evidence-Based Medicine Center, Institute of Translational Medicine Shenzhen University School of Medicine, Shenzhen Second People's Hospital First Affiliated Hospital of Shenzhen University, 3002 Sungang Road, Futian District Shenzhen, Guangdong 518035 (PR China)

E-Mail pupeter190@163.com and lishamou@gmail.com 
copy, stereoscopic colour film fundus photography, slitlamp biomicroscopy, and mydriatic or non-mydriatic digital colour or monochromatic photography [7-10]. The Early Treatment Diabetic Retinopathy Study has documented and established 7-standard-field $35-\mathrm{mm}$ stereoscopic colour retinal imaging (7SF) as the reference standard [11]. Ophthalmologic slit-lamp biomicroscopy is considered sensitive and specific enough for DR screening, diagnosis, and treatment, and it is also an accepted standard reference for DR detection [12]. However, both these methods are considered complex and time-consuming processes, which are not suitable for large-scale DR screening [13]. Therefore, considerable research has focused on the evaluation of a simpler method for diagnosing DR.

Both the UK National Screening Committee and the Health Technology Board for Scotland have recommended a digital camera-based system for DR screening. However, the two groups differ in their recommendations on the use of mydriasis and the number of fields to be used for each eye. The former recommended mydriasis with 2 fields per eye, and the latter advised mydriasis only when undilated photography had failed and recommended the use of a single 45-degree field centred on the macula [14, 15]. The British Diabetic Association recommends that imaging devices in population-based screening should achieve sensitivities of equal to or greater than $80 \%$, while maximizing specificity for referral-warranted DR [16]. Non-mydriatic fundus photography (NMFP) can be used with either pupillary dilation in a dim room or pharmacological dilation; thus, it can be more comfortable for patients and can take less time to perform. These advantages of NMFP make it an efficient and practical tool for use in general clinical practice [17-19]. Single-field NMFP is the simplest and the most accessible method in the primary care and community setting. However, there has been intense debate on whether it has sufficient sensitivity/specificity as a screening tool for DR.

Extensive studies have reported the application of the single-field NMFP, and the conclusions differed between studies. Lin et al. [20] and Aptel et al. [21] reported that a single-field strategy has a sensitivity of $78 \%$ and a specificity of $86 \%$ for referable DR (defined as moderate to severe DR) detection, and a comparable sensitivity and specificity of 77 and 99\%, respectively, for any DR (defined as mild to severe DR) detection. However, Lee et al. [22] reported that screening for DR using 1-field, non-mydriatic, 45-degree digital photography is inadequate with a sensitivity of only 53.8 and $47.6 \%$, respectively. Thus, there is a great need to perform a systematic analysis to determine the efficiency of this strategy in DR screening.
The objective of our study was to perform a meta-analysis to evaluate the diagnostic performance of the singlefield NMFP in detecting DR. Additionally, we conducted a subgroup analysis to compare its efficacy in detecting DR as compared with different reference standards (7SF and slit-lamp biomicroscopy). The results of this review are intended to help clinicians choose the most appropriate and optimal method for DR screening.

\section{Materials and Method}

A systematic review was performed according to the Preferred Reporting Items for Systematic Reviews and Meta-Analyses (PRISMA) guidelines for reporting systematic reviews [23].

\section{Literature Search}

We searched the PubMed, EMBASE, and Cochrane databases for studies published through May 19, 2018. We used a combination of medical subject heading terms and free-text words to define: our population of interest (persons with diabetes); the pathological process of interest (DR); and the specified imaging technique used (fundus photography or digital retinal imaging system). The search strategy was (Fundus Photography OR Digital Retinal Imaging) AND ([[Diabetic Retinopathy [MeSH Terms]] OR Diabetic Retinopathies] OR Diabetic Retinopathy) OR Retinopathy, Diabetic. We also hand searched the references of the articles obtained in full.

\section{Eligibility Criteria}

Two reviewers (Hu J.Y. and Chen R.T.) independently screened all the acquired studies based on information found in the title and abstract. We then evaluated the full text of the selected articles for eligibility. We fully discussed any discrepancy that arose during the study screening and selection process, and it was resolved by consensus.

The inclusion criteria for the study were: prospective studies conducted in patients known or suspected to have DR, the use of a 45-degree non-mydriatic camera with focus on only one centre, the use of 7SF or slit-lamp biomicroscopy as the reference standard, the use of an ophthalmologist to grade images, and the presentation of the original data. The exclusion criteria were: articles on topics not related to our study question (e.g., retinal abnormalities other than DR), retrospective studies, reviews, the inability to extract data.

\section{Data Extraction}

Two reviewers (Hu J.Y. and Chen R.T.) independently extracted data from each study. We jointly discussed and resolved any discrepancies by consensus. The domains of interest in the standardized data extraction were: descriptive and quantitative results (the values of true positive, false positive, false negative, and true negative), population characteristics, and methodological aspects.

\section{Quality Assessment}

Two reviewers (Hu J.Y. and Chen R.T.) independently evaluated the quality of the included studies using the QUADAS-2 method [24]. The QUADAS-2 method considers four main as-
$\mathrm{Hu} / \mathrm{Chen} / \mathrm{Lu} / \mathrm{Dou} / \mathrm{Ye} / \mathrm{Cai} / \mathrm{Pu} / \mathrm{Mou}$ 
Fig. 1. Schematic flow chart of study selec-

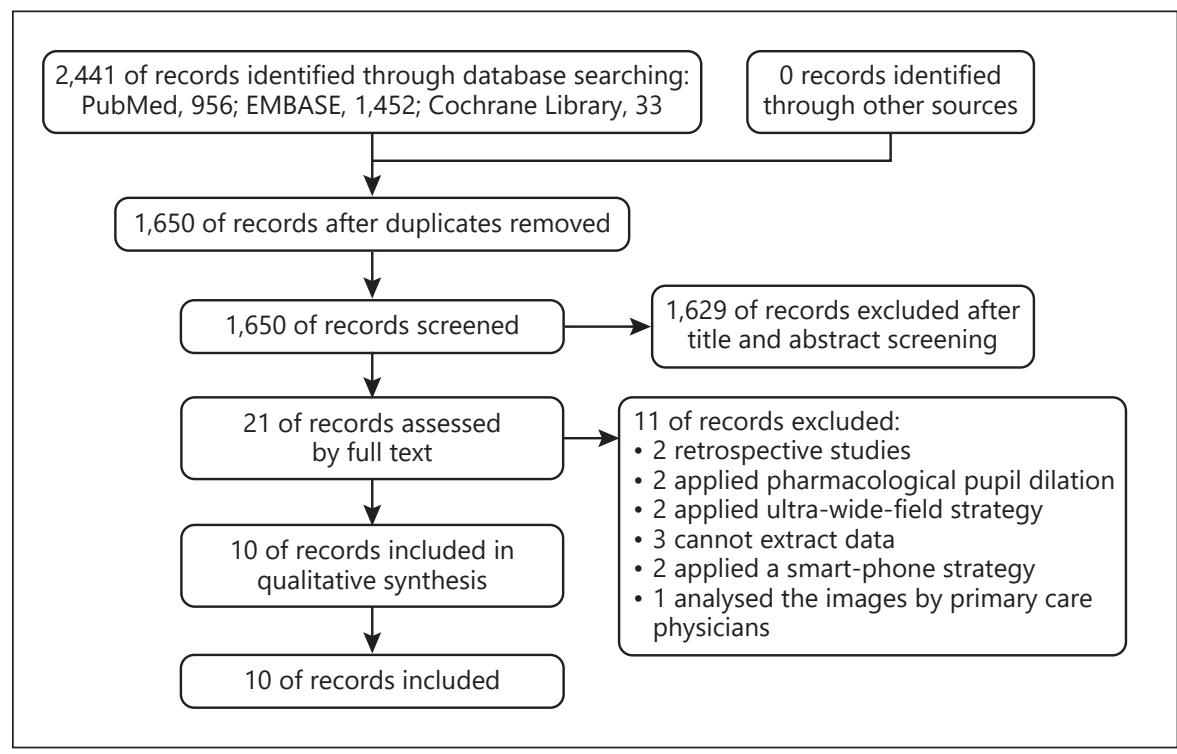
tion of this meta-analysis.

pects for quality assessment: the risk of bias in patient selection, index test, reference test, study flow, and timing. We assessed the risk of bias and applicability concerns for each study; we then evaluated them, using the following assessment: (1) low, if there was a low risk of bias in all key domains; (2) unclear, if we could not assess the risk of bias in one or more key domains; and (3) high, if the risk of bias was high for one or more key domains.

\section{Data Synthesis and Analysis}

The data synthesis and analysis were focused on evaluating the diagnostic performance of the single-field NMFP in detecting any DR. We used the STATA version 12.1 (STATA Corp. LP, College Station, TX, USA), and Review Manager version 5.3 (Computer program, Copenhagen: The Nordic Cochrane Centre, The Cochrane Collaboration) for statistical analyses. The statistical significance was set at $p<0.05$. We calculated a summary estimate of the sensitivity, specificity, positive likelihood ratio (PLR), negative likelihood ratio (NLR), diagnostic odds ratio (DOR), and 95\% confidence intervals (CIs) using a hierarchical random-effects model [25]. We generated the summary receiver-operating characteristic (ROC) curves and documented the sensitivity, specificity, and area under the curve for the index test. If there was no heterogeneity between the studies included in our meta-analysis, the best summary estimate of test performance was designated as a single point on the summary ROC curve (the operating point) [26]. We also assessed statistical heterogeneity among the included studies using the $I^{2}$ statistic, which expresses the percentage of the variability that might result from heterogeneity rather than sampling error. A value of $I^{2}$ greater than the threshold of $50 \%$ was considered as statistical heterogeneity [27]. Funnel plots were created to assess the publication bias by examining the relationship between the effect measure (logDOR) and its standard error [28]. Deeks' test was used to assess asymmetry of the funnel plot and the publication bias, quantitatively [29]. The reference standard was different in the included studies. It was reported by Lawrence [30] that singlefield NMFP had a sensitivity of $66 \%$ when compared to 7 SF and $76 \%$ when compared to dilated clinical examination as the refer-

Effectiveness of Single-Field NMFP in DR Screening ence standard, indicating that the reference applied in the clinical test might have effects on the interpretation of the retinal imaging results. We conducted a subgroup analysis to calculate the sensitivity and specificity according to the reference standard used in the included studies.

\section{Results}

\section{Screening and Selection of Literature}

Our search of the PubMed, EMBASE, and Cochrane databases identified 1,650 articles after the duplicates had been removed. After screening each article based on its title and abstract, we finally assessed the full text of 21 studies and included 10 studies in our meta-analysis [20$22,30-36]$. All of the 21 studies were available as full text. The selection process is shown in Figure 1.

\section{Characteristics and Methodological Aspects of the Included Studies}

The number of the included subjects, age and gender, disease type, and disease duration of the patients of each study were summarized in Table 1 . As shown in Table 1, among the 10 included studies, 7 used 7SF as the reference standard, and 3 used slit-lamp biomicroscopy. The camera brands included Canon, Topcon, and Sony. The focus centre varied in different studies, including the optic disc, the macula, and horizontally and vertically on a point halfway between the temporal edge of the optic disc and the fovea. The definition of DR used in the selected studies did not include diabetic macular oedema. We assessed the methodological quality and risk of bias of the included studies 
Table 1. Characteristics of the included studies

a Study design and patients

\begin{tabular}{|c|c|c|c|c|}
\hline Study ID & $\begin{array}{l}\text { Subjects/ } \\
\text { M }\end{array}$ & Mean age, years & Disease type & $\begin{array}{l}\text { Duration of disease, } \\
\text { years }\end{array}$ \\
\hline Aptel [21], 2008 & $79 / 47 \%$ & 52.4 & type I and II & $4.3-8.9$ \\
\hline Baeza [32], 2009 & $216 / 43.7 \%$ & 68.5 & type I and II & 12.8 \\
\hline Herbert [34], 2003 & $145 / \mathrm{NA}$ & NA & type I and II & NA \\
\hline Lawrence [30], 2004 & $103 / 98.1 \%$ & 67.5 & any type of diabetes & 12.4 \\
\hline Lee [22], 2011 & $303 / 63.2 \%$ & 53.1 & type I and II & 5.4 \\
\hline Lin [20], 2002 & $197 / 58 \%$ & $21-80+$ & type I and II & any disease duration \\
\hline Phiri [35], 2006 & $196 / 57 \%$ & 68.5 & most were type II & 12.3 \\
\hline Pugh [36], 1993 & $352 / 76 \%$ & $59.7 \%$ under 59 & IDDM and NIDDM & 9.8 \\
\hline Taylor [31], 1999 & $118 / 44 \%$ & $40-80+$ & any type of diabetes & $0-20+$ \\
\hline Vujosevic [33], 2009 & $55 / 60 \%$ & 57.1 & type I and II & type I, 23.3; type II, 15.8 \\
\hline \multicolumn{5}{|c|}{ b Reference and index test of studies } \\
\hline Study ID & $\begin{array}{l}\text { Reference } \\
\text { test }\end{array}$ & Cameras and size of field & Pixels & Focus centre \\
\hline Aptel [21], 2008 & slit-lamp & Topcon TRC-NW6S, $45^{\circ}$ & $800 \times 600$ & fovea \\
\hline Baeza [32], 2009 & $7 \mathrm{SF}$ & Topcon CRW6, $45^{\circ}$ & NA & macula \\
\hline Herbert [34], 2003 & slit-lamp & Topcon TRC-NW5S, $45^{\circ}$ & $800 \times 600$ & macula \\
\hline Lawrence [30], 2004 & $7 \mathrm{SF}$ & Topcon TRC-NW6S, $45^{\circ}$ & $640 \times 480$ & optical disc \\
\hline Lee [22], 2011 & slit-lamp & Topcon TRC-NW5S, $45^{\circ}$ & $800 \times 600$ & optical discs \\
\hline Lin $[20], 2002$ & $7 \mathrm{SF}$ & Canon CR5-45NM, $45^{\circ}$ & $640 \times 480$ & $\begin{array}{l}\text { mid between fovea and } \\
\text { optic disc and the fovea }\end{array}$ \\
\hline Phiri [35], 2006 & $7 \mathrm{SF}$ & Canon CR6 45NM, $45^{\circ}$ & $2,160 \times 1,440$ & $\begin{array}{l}\text { mid between macula of } \\
\text { optic disc }\end{array}$ \\
\hline Pugh [36], 1993 & $7 \mathrm{SF}$ & Canon CR3 45NM, $45^{\circ}$ & NA & $\begin{array}{l}\text { mid between fovea and } \\
\text { optic disc }\end{array}$ \\
\hline Taylor [31], 1999 & $7 \mathrm{SF}$ & Topcon $45 \mathrm{NM}, 45^{\circ}$ & $640 \times 480$ & $\begin{array}{l}\text { mid between macula } \\
\text { and optic disc }\end{array}$ \\
\hline Vujosevic [33], 2009 & $7 \mathrm{SF}$ & $\begin{array}{l}\text { Nidek NM retinography } \\
\text { device, } 45^{\circ}\end{array}$ & $1,392 \times 1,040$ & central field \\
\hline
\end{tabular}

M, male; NA, not available; IDDM, insulin-dependent diabetes mellitus; NIDDM, non-insulin-dependent diabetes mellitus; 7SF, 7-standard-field 35-mm stereoscopic colour retinal image.

based on the QUADAS-2 checklist (patient selection, index test, reference standard, flow, and timing). The results are shown in online supplementary Figure 1 (for all online suppl. material, see www.karger.com/doi/10.1159/000499106).

\section{Diagnostic Performance of Single-Field NMFP}

Pooled diagnostic indicators including sensitivity, specificity, PLR, NLR, and DOR of single-field NMFP for any DR were calculated. As shown in Table 2 and online supplementary Figure 2, the overall sensitivity and specificity were 68\% (95\% CI: 59-76) and 94\% (95\% CI: 0.89-0.97), respectively; additionally, the PLR and NLR were 11.2 (95\% CI: 6.1-20.8) and 0.34 (95\% CI: 0.26-0.44), respectively.
The pooled DOR estimate, using the random-effects model, was 33 (95\% CI: 17-65). Figure 2 presents the summary ROC curve based on a bivariate model. The expected operating point on the summary ROC corresponded to a sensitivity of 0.68 (95\% CI: $0.59-0.76)$ and a specificity of 0.94 (95\% CI: 0.89-0.97). The summary AUC value was 0.88 (95\% CI: 0.85-0.90). We also assessed the heterogeneity. As shown in online supplementary Figure 2, the value of $I^{2}$ of sensitivity and specificity were 87 and 95\%, respectively.

\section{Subgrouping Analysis}

We classified the studies into subgroups according to the reference standard used in the diagnostic studies. As 
Table 2. Estimates of the diagnostic indicators of included studies a Pooled estimates of the included studies

\begin{tabular}{lcc}
\hline Parameter & Estimate & $95 \%$ CI \\
\hline Sensitivity & 0.68 & $0.59-0.76$ \\
Specificity & 0.94 & $0.89-0.97$ \\
PLR & 11.2 & $6.1-20.8$ \\
NLR & 0.34 & $0.26-0.44$ \\
DOR & 33 & $17-65$ \\
\hline
\end{tabular}

b Estimates of the subgrouping studies

\begin{tabular}{lll}
\hline Reference & Sensitivity & Specificity \\
\hline 7SF & $0.73(0.66-0.81)$ & $0.91(0.85-0.97)$ \\
Slit-lamp biomicroscopy & $0.54(0.39-0.70)$ & $0.98(0.95-1.00)$ \\
\hline
\end{tabular}

CI, confidence interval: PLR, positive likelihood ratio; NLR, negative likelihood ratio; DOR, diagnostic odds ratio; 7SF, 7-standard-field 35-mm stereoscopic colour retinal image.

shown in Table 2, the sensitivity and specificity were 0.73 (95\% CI: 0.66-0.81) and 0.91 (95\% CI: 0.85-0.97), respectively, when compared to 7SF; they were 0.54 (95\% CI: $0.39-0.70)$ and 0.98 (95\% CI: 0.95-1.00), respectively, when compared to slit-lamp biomicroscopy.

\section{Publication Bias}

The linear regression test of funnel plot asymmetry can be established with more than two sets of data. As shown in online supplementary Figure 3, there was no evidence of publication bias among the included studies with a $p$ value of 0.39 using Deeks' test.

\section{Discussion}

Information on the presence and the level of DR is useful when making therapeutic decisions or predicting disease outcomes. NMFP has been widely used in DR screening, and most organizations and consensus have recommended a 2-field strategy [37, 38]. It is unknown whether the simpler single-field strategy is efficient and meets standards for DR detection. This meta-analysis documented the performance of single-field NMFP in DR detection, in an effort to evaluate its diagnostic accuracy as a potentially new standard method.

The results of our meta-analysis showed that 45-degree, single-field NMFP has a pooled sensitivity and specificity of 68 and $94 \%$, respectively. Sensitivity values

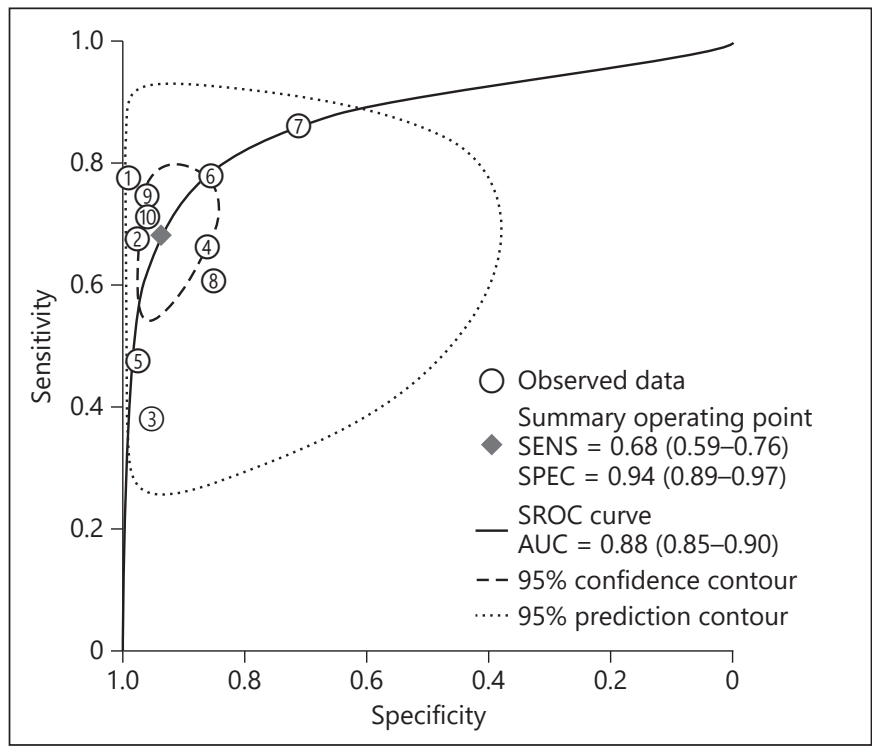

Fig. 2. ROC curves of all studies which were included in the metaanalysis. The dotted lines are the $95 \%$ prediction intervals calculated using a bivariate hierarchical model with the STATA-12.1 program. SENS, sensitivity; SPEC, specificity; SROC, summary receiver-operating characteristic; AUC, area under the curve.

of $80 \%$ and specificity values of $95 \%$, combined with a technical failure rate of less than $5 \%$, are considered the standard values in retinopathy detection, as proposed by the British Diabetic Association [16]. In this regard, single-field NMFP does not meet the technical requirements in DR screening and is thus not an ideal clinical practice. Since the grading criteria for "referable DR" differ in the included studies, we are unable to estimate the diagnostic performance for referable DR. It would be interesting to estimate the diagnostic performance of single-field NMFP for referable DR in the future with the same grading criteria. The subgroup analysis demonstrated the sensitivity and specificity of single-field NMFP were 0.73 and 0.91 when compared with 7SF photography, and they were 0.54 and 0.98 when compared with slit-lamp biomicroscopy, suggesting that the chosen reference standard may have an effect on the interpretation of the fundus photography images. Some studies have suggested that dilated fundus photography is more sensitive than slit-lamp biomicroscopy [30], so clinical examination by slit-lamp biomicroscopy versus the 7-field, 30-degree mydriatic retinal photography, which is the best reference standard? Our meta-analysis just included three studies that used slit-lamp biomicroscopy as reference standard, and more research in the future is needed to get definitive conclusions. 
The pooled sensitivity of single-field NMFP was only 0.68 in any DR detection, meaning that about one third of the patients with minimal lesions may go undetected. Failure to detect microhaemangioma and retinopathy outside the single 45 -degree image may be the main cause, as several studies have suggested that multiple field and ultra-wide field imaging had a much better performance in DR detection [38-40]. Additionally, the use of mydriasis was reported to reduce the proportion of ungradable photographs [41]. The addition of a nasal image, ultrawide field image, and the application of pharmacological pupil dilation could be helpful in improving the diagnostic performance of retinal imaging.

The results of this meta-analysis suggested significant heterogeneity among the included studies. The heterogeneity has multiple sources, including the characteristics of patients imaged, the differences in the imaging procedure, the qualities of the pictures, the experience of the ophthalmologist, differences in the reference test, the interpretation criteria, or a combination of these factors. Our subgroup analysis showed the sensitivity of single-filed NMFP varied significantly when compared with different reference standards, suggesting that the reference standard applied in each single study may also be the cause of heterogeneity.

Our study had limitations. First, there were only 10 articles with a relatively small number of subjects who met our inclusion criteria for the final meta-analysis. Second, the summary results of this meta-analysis had high statistical heterogeneity. Third, both randomized control and observational studies were included in this meta-analysis. Although only randomized, controlled studies are preferable, a systematic review including both randomized and observational studies provided a tool for synthesizing clinical data when randomized, controlled studies were limited $[42,43]$. Additionally, a meta-analytic pooled estimate from the primary articles would be inappropriate because of the risk of bias in the included studies [44].
In conclusion, our systematic review and meta-analysis suggested that single-field retinal imaging is insufficient to detect any DR. Additionally, different reference standards used in the diagnosis process could affect the final diagnostic results. Selecting the most appropriate test in any clinical situation depends on the particular circumstances of the patient, the expertise of the clinician, availability of equipment, and the costs of the procedures. The goal is to use the most cost-effective imaging to allow accurate diagnosis and prompt treatment of this common, complex, and costly problem. However, this will require further prospective studies with larger numbers of patients and with a direct comparison between the different diagnostic techniques.

\section{Statement of Ethics}

The authors have no ethical conflicts to disclose.

\section{Disclosure Statement}

The authors have no conflicts of interest to declare.

\section{Funding Sources}

This work was supported in part by grants from the National Key R\&D Program of China (2017YFC1103704), Sanming Project of Medicine in Shenzhen (SZSM201412020), Fund for High Level Medical Discipline Construction of Shenzhen (2016031638), and the Shenzhen Foundation of Health and Family Planning Commission (SZXJ2017021).

\section{Author Contributions}

Study planning: Zuhui Pu, Lisha Mou, Zhiming Cai. Searched literature and extracted data: Jiying $\mathrm{Hu}$ and Ruiting Chen. Analysed the data: Jiying Hu, Ying Lu, Baikang Ye, Xiaoyan Dou. Wrote the paper: Jiying $\mathrm{Hu}$, Lisha Mou.

\section{References}

1 Collaboration NC; NCD Risk Factor Collaboration (NCD-RisC). Worldwide trends in diabetes since 1980: a pooled analysis of 751 population-based studies with 4.4 million participants. Lancet. 2016 Apr;387(10027):1513-30.

2 Cho NH, Kirigia J, Mbanya JC, Ogurstova K, Guariguata L, Rathmann W, et al. IDF diabetes atlas. 8th ed. 2017. Available from: http:// www.diabetesatlas.org/resources/2017-atlas. html
3 Leasher JL, Bourne RR, Flaxman SR, Jonas JB, Keeffe J, Naidoo K, et al; Vision Loss Expert Group of the Global Burden of Disease Study. Global estimates on the number of people blind or visually impaired by diabetic retinopathy: a meta-analysis from 1990 to 2010. Diabetes Care. 2016 Sep;39(9):1643-9.

4 Rosenberg JB, Tsui I. Screening for Diabetic Retinopathy. N Engl J Med. 2017 Apr;376(16): 1587-8.
5 Lamoureux EL, Taylor H, Wong TY. Frequency of Evidence-Based Screening for Diabetic Retinopathy. N Engl J Med. 2017 Jul; 377(2):194-5.

6 Nguyen HV, Tan GS, Tapp RJ, Mital S, Ting DS, Wong HT, et al. Cost-effectiveness of a National Telemedicine Diabetic Retinopathy Screening Program in Singapore. Ophthalmology. 2016 Dec;123(12):2571-80. 
7 Ahmed J, Ward TP, Bursell SE, Aiello LM, Cavallerano JD, Vigersky RA. The sensitivity and specificity of nonmydriatic digital stereoscopic retinal imaging in detecting diabetic retinopathy. Diabetes Care. 2006 Oct;29(10):2205-9.

8 Wang S, Zuo Y, Wang N, Tong B. Fundus fluorescence Angiography in diagnosing diabetic retinopathy. Pak J Med Sci. 2017 Nov-Dec; 33(6):1328-32.

9 Virgili G, Menchini F, Dimastrogiovanni AF, Rapizzi E, Menchini U, Bandello F, et al. Optical coherence tomography versus stereoscopic fundus photography or biomicroscopy for diagnosing diabetic macular edema: a systematic review. Invest Ophthalmol Vis Sci. 2007 Nov;48(11):4963-73.

10 Henricsson M, Karlsson C, Ekholm L, Kaikkonen P, Sellman A, Steffert E, et al. Colour slides or digital photography in diabetes screening-a comparison. Acta Ophthalmol Scand. 2000 Apr;78(2):164-8.

11 Bursell SE, Cavallerano JD, Cavallerano AA, Clermont AC, Birkmire-Peters D, Aiello LP, et al.; Joslin Vision Network Research Team. Stereo nonmydriatic digital-video color retinal imaging compared with Early Treatment Diabetic Retinopathy Study seven standard field $35-\mathrm{mm}$ stereo color photos for determining level of diabetic retinopathy. Ophthalmology. 2001 Mar;108(3):572-85.

12 Prasad S, Kamath GG, Jones K, Clearkin LG, Phillips RP. Effectiveness of optometrist screening for diabetic retinopathy using slitlamp biomicroscopy. Eye (Lond). 2001 Oct; 15(Pt 5):595-601.

13 George LD, Halliwell M, Hill R, Aldington SJ, Lusty J, Dunstan F, et al. A comparison of digital retinal images and $35 \mathrm{~mm}$ colour transparencies in detecting and grading diabetic retinopathy. Diabet Med. 1998 Mar;15(3): 250-3.

14 Scanlon PH. The English National Screening Programme for diabetic retinopathy 20032016. Acta Diabetol. 2017 Jun;54(6):515-25.

15 NHS Scotland. Scottish diabetic retinopathy screening collaborative [online]. 2015. Available from: http://www.ndrs-wp.scot.nhs.uk/

16 British Diabetic Association. Retinal Photography Screening for Diabetic Eye Disease. London: British Diabetic Association; 1997.

$17 \mathrm{Ku} \mathrm{JJ}$, Landers J, Henderson T, Craig JE. The reliability of single-field fundus photography in screening for diabetic retinopathy: the Central Australian Ocular Health Study. Med J Aust. 2013 Feb;198(2):93-6.

18 Diamond JP, McKinnon M, Barry C, Geary D, McAllister IL, House P, et al. Non-mydriatic fundus photography: a viable alternative to fundoscopy for identification of diabetic retinopathy in an Aboriginal population in rural Western Australia? Aust N Z J Ophthalmol. 1998 May;26(2):109-15.

19 Farley TF, Mandava N, Prall FR, Carsky C. Accuracy of primary care clinicians in screening for diabetic retinopathy using single-image retinal photography. Ann Fam Med. 2008 Sep-Oct;6(5):428-34.
20 Lin DY, Blumenkranz MS, Brothers RJ, Grosvenor $\mathrm{DM}$. The sensitivity and specificity of single-field nonmydriatic monochromatic digital fundus photography with remote image interpretation for diabetic retinopathy screening: a comparison with ophthalmoscopy and standardized mydriatic color photography. Am J Ophthalmol. 2002 Aug;134(2): 204-13.

21 Aptel F, Denis P, Rouberol F, Thivolet C. Screening of diabetic retinopathy: effect of field number and mydriasis on sensitivity and specificity of digital fundus photography. Diabetes Metab. 2008 Jun;34(3):290-3.

22 Lee DW, Bae JH, Song SJ. Efficacy of singlefield non-mydriatic digital fundus photography for screening diabetic retinopathy. J Korean Ophthalmol Soc. 2011 May;52(5):531-6.

23 Moher D, Liberati A, Tetzlaff J, Altman DG; PRISMA Group. Preferred reporting items for systematic reviews and meta-analyses: the PRISMA statement. Ann Intern Med. 2009 Aug;151(4):264-9.

24 Whiting PF, Rutjes AW, Westwood ME, Mallett S, Deeks JJ, Reitsma JB, et al.; QUADAS-2 Group. QUADAS-2: a revised tool for the quality assessment of diagnostic accuracy studies. Ann Intern Med. 2011 Oct;155(8): 529-36.

25 Foxlee N, Stone JC, Doi SA. A comparison of univariate and bivariate models in meta-analysis of diagnostic accuracy studies. Int J Evid Based Healthc. 2015 Mar;13(1):28-34.

26 Harbord RM, Deeks JJ, Egger M, Whiting P, Sterne JA. A unification of models for metaanalysis of diagnostic accuracy studies. Biostatistics. 2007 Apr;8(2):239-51.

27 Koletsi D, Fleming PS, Michelaki I, Pandis N. Heterogeneity in Cochrane and non-Cochrane meta-analyses in orthodontics. J Dent. 2018 Jul; $74: 90-4$.

28 Hunter JP, Saratzis A, Sutton AJ, Boucher RH, Sayers RD, Bown MJ. In meta-analyses of proportion studies, funnel plots were found to be an inaccurate method of assessing publication bias. J Clin Epidemiol. 2014 Aug;67(8):897-903.

29 Lin L, Chu H, Murad MH, Hong C, Qu Z, Cole SR, et al. Empirical Comparison of Publication Bias Tests in Meta-Analysis. J Gen Intern Med. 2018 Aug;33(8):1260-7.

30 Lawrence MG. The accuracy of digital-video retinal imaging to screen for diabetic retinopathy: an analysis of two digital-video retinal imaging systems using standard stereoscopic seven-field photography and dilated clinical examination as reference standards. Trans Am Ophthalmol Soc. 2004;102:321-40.

31 Taylor DJ, Fisher J, Jacob J, Tooke JE. The use of digital cameras in a mobile retinal screening environment. Diabet Med. 1999 Aug; 16(8):680-6.

32 Baeza M, Orozco-Beltrán D, Gil-Guillen VF, Pedrera V, Ribera MC, Pertusa S, et al. Screening for sight threatening diabetic retinopathy using non-mydriatic retinal camera in a primary care setting: to dilate or not to dilate? Int J Clin Pract. 2009 Mar;63(3):433-8.
33 Vujosevic S, Benetti E, Massignan F, Pilotto E, Varano M, Cavarzeran F, et al. Screening for diabetic retinopathy: 1 and 3 nonmydriatic 45-degree digital fundus photographs vs 7 standard early treatment diabetic retinopathy study fields. Am J Ophthalmol. 2009 Jul; 148(1):111-8.

34 Herbert HM, Jordan K, Flanagan DW. Is screening with digital imaging using one retinal view adequate? Eye (Lond). 2003 May; 17(4):497-500.

35 Phiri R, Keeffe JE, Harper CA, Taylor HR Comparative study of the polaroid and digital non-mydriatic cameras in the detection of referrable diabetic retinopathy in Australia. Diabet Med. 2006 Aug;23(8):867-72.

36 Pugh JA, Jacobson JM, Van Heuven WA, Watters JA, Tuley MR, Lairson DR, et al. Screening for diabetic retinopathy. The wideangle retinal camera. Diabetes Care. 1993 Jun; 16(6):889-95.

37 Hansen AB, Hartvig NV, Jensen MS, BorchJohnsen K, Lund-Andersen H, Larsen M. Diabetic retinopathy screening using digital non-mydriatic fundus photography and automated image analysis. Acta Ophthalmol Scand. 2004 Dec;82(6):666-72.

38 Ghasemi Falavarjani K, Tsui I, Sadda SR. Ultra-wide-field imaging in diabetic retinopathy. Vision Res. 2017 Oct;139:187-90.

39 Hirano T, Imai A, Kasamatsu H, Kakihara S, Toriyama Y, Murata T. Assessment of diabetic retinopathy using two ultra-wide-field fundus imaging systems, the Clarus ${ }^{\circledR}$ and Optos $^{\mathrm{TM}}$ systems. BMC Ophthalmol. 2018 Dec; 18(1):332.

40 Srihatrai $\mathrm{P}$, Hlowchitsieng T. The diagnostic accuracy of single- and five-field fundus photography in diabetic retinopathy screening by primary care physicians. Indian J Ophthalmol. 2018 Jan;66(1):94-7.

41 Banaee T, Ansari-Astaneh MR, Pourreza H, Faal Hosseini F, Vatanparast M, Shoeibi N, et al. Utility of $1 \%$ Tropicamide in Improving the Quality of Images for Tele-Screening of Diabetic Retinopathy in Patients with Dark Irides. Ophthalmic Epidemiol. 2017 Aug; 24(4):217-21.

42 Kriston L. Dealing with clinical heterogeneity in meta-analysis. Assumptions, methods, interpretation. Int J Methods Psychiatr Res. 2013 Mar;22(1):1-15.

43 Meybohm P, Choorapoikayil S, Wessels A, Herrmann E, Zacharowski K, Spahn DR. Washed cell salvage in surgical patients: A review and meta-analysis of prospective randomized trials under PRISMA. Medicine (Baltimore). 2016 Aug;95(31):e4490.

44 Scargle JD. Publication bias: the "file-drawer" problem in scientific inference. J Sci Explor. 2000 Sep;14(1):91-106. 